\title{
EDITORIAL
}

\section{Artificial neural networks in medical diagnosis}

\author{
Filippo Amato ${ }^{1}$, Alberto López ${ }^{1 *}$, Eladia María Peña-Méndez ${ }^{2}$, Petr Vaňhara ${ }^{3}$, Aleš Hampl ${ }^{3,4}$, \\ Josef Havel ${ }^{1,5,6}$ \\ ${ }^{1}$ Department of Chemistry, Faculty of Science, Masaryk University, Brno, Czech Republic \\ ${ }^{2}$ Department of Analytical Chemistry, Nutrition and Food Science, Faculty of Chemistry, University of La Laguna, \\ La Laguna, Tenerife, Spain \\ ${ }^{3}$ Department of Histology and Embryology, Faculty of Medicine, Masaryk University, Brno, Czech Republic \\ ${ }^{4}$ International Clinical Research Center, St. Anne's University Hospital, Brno, Czech Republic \\ ${ }^{5}$ Department of Physical Electronics, Faculty of Science, Masaryk University, Brno, Czech Republic \\ ${ }^{6} \mathrm{R} \& \mathrm{D}$ Centre for low-cost plasma and nanotechnology surface modifications, CEPLANT, Masaryk University, \\ Brno, Czech Republic \\ * On leave from the University of Salamanca, Spain
}

Received $17^{\text {th }}$ December 2012.

Published online $7^{\text {th }}$ January 2013.

\begin{abstract}
Summary
An extensive amount of information is currently available to clinical specialists, ranging from details of clinical symptoms to various types of biochemical data and outputs of imaging devices. Each type of data provides information that must be evaluated and assigned to a particular pathology during the diagnostic process. To streamline the diagnostic process in daily routine and avoid misdiagnosis, artificial intelligence methods (especially computer aided diagnosis and artificial neural networks) can be employed. These adaptive learning algorithms can handle diverse types of medical data and integrate them into categorized outputs. In this paper, we briefly review and discuss the philosophy, capabilities, and limitations of artificial neural networks in medical diagnosis through selected examples.
\end{abstract}

Key words: medical diagnosis; artificial intelligence; artificial neural networks; cancer; cardiovascular diseases; diabetes

\section{INTRODUCTION}

Artificial neural networks (ANNs) are widely used in science and technology with applications in various branches of chemistry, physics, and biology.

Josef Havel, Department of Chemistry, Faculty of Science, Masaryk University, Kamenice 5/ A14, 62500 Brno, Czech Republic

ㅁ. havel@chemi.muni.cz

용 +420549494114

圆 +420549492494

(C) Journal of Applied Biomedicine
For example, ANNs are used in chemical kinetics (Amato et al. 2012), prediction of the behavior of industrial reactors (Molga et al. 2000), modeling kinetics of drug release (Li et al. 2005), optimization of electrophoretic methods (Havel et al. 1998), classification of agricultural products such as onion varieties (Rodríguez Galdón et al. 2010), and even species determination (Fedor et al. 2008, Michalkova et al. 2009, Murarikova et al. 2010). In general, very diverse data such as classification of biological objects, chemical kinetic data, or even clinical parameters can be handled in essentially the same way. Advanced computational methods, including ANNs, utilize diverse types of input data that are processed in the context of previous training history 
on a defined sample database to produce a clinically relevant output, for example the probability of a certain pathology or classification of biomedical objects. Due to the substantial plasticity of input data, ANNs have proven useful in the analysis of blood and urine samples of diabetic patients (Catalogna et al. 2012, Fernandez de Canete et al. 2012), diagnosis of tuberculosis (Er et al. 2008, Elveren and Yumușak 2011), leukemia classification (Dey et al. 2012), analysis of complicated effusion samples (Barwad et al. 2012), and image analysis of radiographs or even living tissue (Barbosa et al. 2012, Saghiri et al. 2012).

The aim of this paper is to present the general philosophy for the use of ANNs in diagnostic approaches through selected examples, documenting the enormous variability of data that can serve as inputs for ANNs. Attention will not only be given to the power of ANNs applications, but also to evaluation of their limits, possible trends, and future developments and connections to other branches of human medicine (Fig. 1).

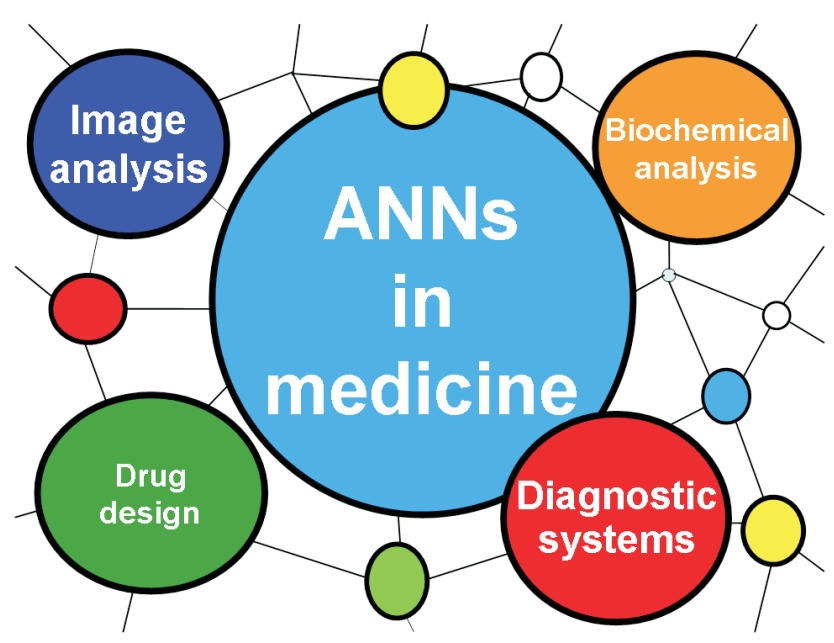

Fig. 1. Overview of the main applications of artificial neural networks in medicine.

\section{ARTIFICIAL NEURAL NETWORKS}

An ANN is a mathematical representation of the human neural architecture, reflecting its "learning" and "generalization" abilities. For this reason, ANNs belong to the field of artificial intelligence. ANNs are widely applied in research because they can model highly non-linear systems in which the relationship among the variables is unknown or very complex. A review of various classes of neural networks can be found in (Aleksander and Morton 1995, Zupan and Gasteiger 1999).

\section{Mathematical background}

A neural network is formed by a series of "neurons" (or "nodes") that are organized in layers. Each neuron in a layer is connected with each neuron in the next layer through a weighted connection. The value of the weight $\mathrm{w}_{i j}$ indicates the strength of the connection between the $i^{- \text {th }}$ neuron in a layer and the $j^{\text {th }}$ neuron in the next one.
The structure of a neural network is formed by an "input" layer, one or more "hidden" layers, and the "output" layer. The number of neurons in a layer and the number of layers depends strongly on the complexity of the system studied. Therefore, the optimal network architecture must be determined. The general scheme of a typical three-layered ANN architecture is given in Fig. 2.

The neurons in the input layer receive the data and transfer them to neurons in the first hidden layer through the weighted links. Here, the data are mathematically processed and the result is transferred to the neurons in the next layer. Ultimately, the neurons in the last layer provide the network's output. The $j$-th neuron in a hidden layer processes the incoming data $\left(x_{i}\right)$ by: $(i)$ calculating the weighted sum and adding a "bias" term $\left(\theta_{j}\right)$ according to Eq. 1:

$$
\text { net }_{j}=\sum_{i=1}^{m} x_{i} \times w_{i j}+\theta_{j} \quad(j=1,2, \ldots, n)
$$




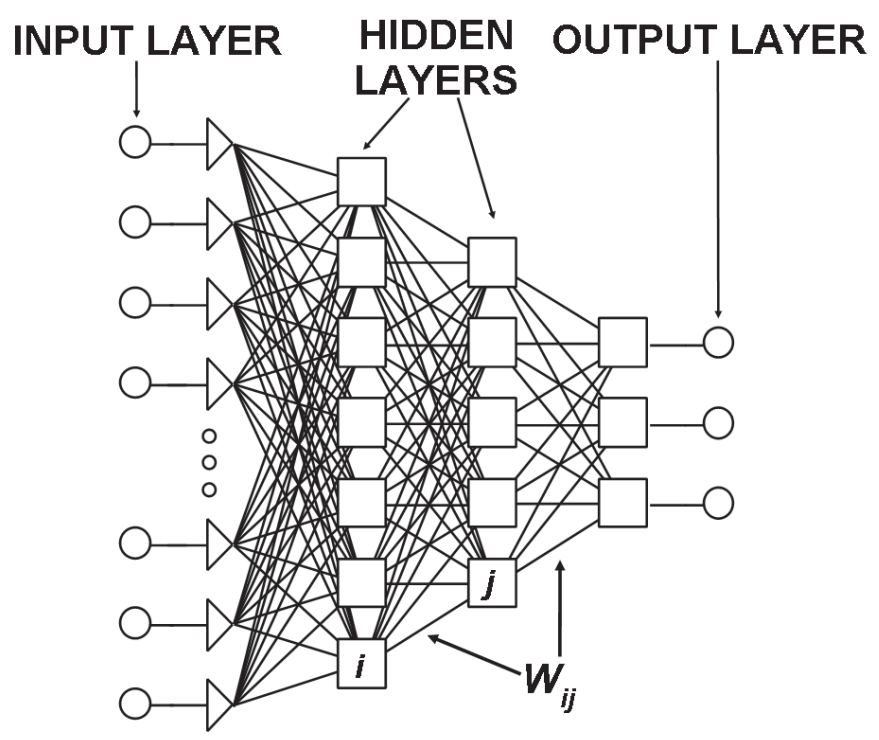

Fig. 2. General structure of a neural network with two hidden layers. The $w_{i j}$ is the weight of the connection between the $i^{\text {th }}$ and the $j^{- \text {th }}$ node.

(ii) transforming the net through a suitable mathematical "transfer function", and (iii) transferring the result to neurons in the next layer. Various transfer functions are available (Zupan and Gasteiger 1999); however, the most commonly used is the sigmoid one:

$$
f(x)=\frac{1}{1+e^{-x}}
$$

\section{Network learning}

The mathematical process through which the network achieves "learning" can be principally ignored by the final user. In this way, the network can be viewed as a "black box" that receives a vector with $m$ inputs and provides a vector with $n$ outputs (Fig. 3). Here we will give only a brief description of the learning process; more details are provided for example in the review by (Basheer and Hajmeer 2000). The network "learns" from a series of "examples" that form the "training database" (Fig. 4). An "example" is formed by a vector $X_{i m}=\left(x_{i l}, x_{i 2}, \ldots ., x_{i m}\right)$ of inputs and a vector $Y_{i n}=\left(y_{i 1}, y_{i 2}, \ldots ., y_{i n}\right)$ of outputs. The objective of the training process is to approximate the function $f$ between the vectors $X_{i m}$ and the $Y_{i n}$ :

$$
Y_{i, n}=f\left(X_{i, m}\right)
$$

This is achieved by changing iteratively the values of the connection weights $\left(w_{i j}\right)$ according to a suitable mathematical rule called the training algorithm.
The values of the weights are changed by using the steepest descent method to minimize a suitable function used as the training stopping criteria. One of the functions most commonly used is the sum-ofsquared residuals given by Eq. 4 :

$$
E=\frac{1}{2} \sum_{i=1}^{m} \sum_{j=1}^{n}\left(y_{i j}-y_{i j}^{*}\right)^{2}
$$

where $y_{i j}$ and $y_{i j}{ }^{*}$ are the actual and network's $j{ }^{\text {th }}$ output corresponding to the $i^{\text {th }}$ input vector, respectively.

The current weight change on a given layer is given by Eq. (5):

$$
\Delta w_{i j}=-\eta \frac{d E}{d w_{i j}}
$$

where $\eta$ is a positive constant called the learning rate. To achieve faster learning and avoid local minima, an additional term is used and Eq. 5 becomes:

$$
\Delta w_{i j}^{k}=-\eta \frac{d E}{d w_{i j}}+\mu \Delta w_{i j}^{k-1}
$$

where $\mu$ is the "momentum" term and $\Delta w_{i j}^{k-1}$ is the change of the weight $w_{i j}$ from the $(k-1)$ - $^{\text {th }}$ learning cycle. The learning rate controls the weight update rate according to the new weight change and the momentum acts as a stabilizer, being aware of the previous weight change. 


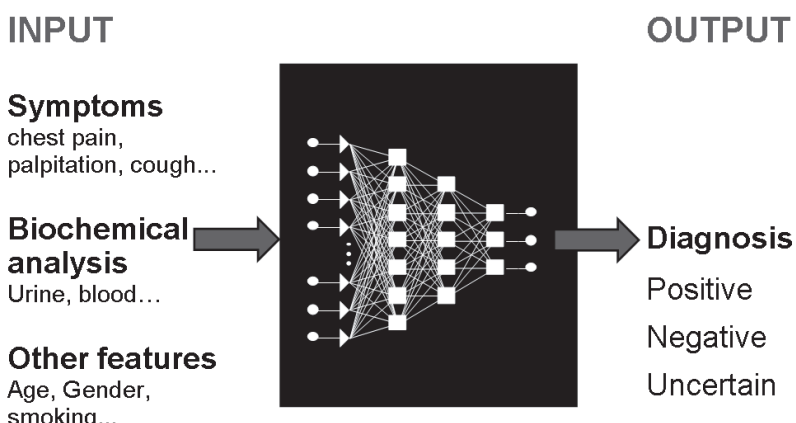

Fig. 3. Details of input and output items concerning ANNs-based diagnosis (ANN architecture is often hidden and it is indicated here as a black box).

\begin{tabular}{|c|c|c|}
\hline $\begin{array}{l}\text { Patien } \\
\text { code }\end{array}$ & MEDICAL DATA & DIAGNOSIS \\
\hline 1 & data $_{1,1} \ldots \quad$ data $_{1, i} \ldots \quad$ data $_{1, m}$ & POSITIVE \\
\hline 2 & data $_{2,1} \ldots$ data $_{2, i} \ldots \quad$ data $_{2, m}$ & POSITIVE \\
\hline 3 & data $_{3,1} \ldots$ data $_{3, i} \ldots \quad$ data $_{3, m}$ & POSITIVE \\
\hline$k$ & $\operatorname{data}_{k, 1} \ldots$ data $_{k, i} \ldots$ data $_{k, m}$ & NEGATIVE \\
\hline$k+1$ & data $_{k+1,1} \ldots$ data $a_{k+1, i} \ldots$ data $_{k+1, m}$ & $m$ NEGATIVE \\
\hline & data $_{n, 1} \ldots \quad$ data $_{n, i} \ldots \quad$ data $_{n, m}$ & NEGATIVE \\
\hline
\end{tabular}

Fig. 4. Example of training database structure. Each row refers to a different patient labeled with a numerical code. The element data $a_{k, i}$ refers to the $i^{- \text {th }}$ medical data (symptom, laboratory data, etc.) of the $k^{\text {-th }}$ patient.

The function given by Eq. 4 is also used as the criterion to optimize the network architecture because it depends on the number of hidden layers and the number of neurons therein. To find the optimal architecture, the most common approach is to plot the value of $E$ (Eq. 4) as a function of the number of nodes in the hidden layer $(q)$. An example of such a plot is given in Fig. 5. As $q$ increases, $E$ decreases. However, after an optimal value of $q$ the improvement is rather poor. Usually, the optimal value of $q$ is found from the intersection point of the two branches of the plot.

After the optimal neural network architecture is found, the training process is performed until a proper minimum value of $E$ is reached. Afterward, the network is checked with examples not previously used in the training step. This process is called verification. Finally, the network can be used to predict outputs for new input vectors.
Structure of the training database

As stated above, the network must be trained using a suitable database. The database is a table (or matrix) of data concerning patients for whom the diagnosis (positive or negative) about a certain disease is already known. Each row of the matrix refers to one patient. The first $m$ elements of the row are medical data and the last $n$ elements represent the output (diagnosis). The term "medical data" indicates biochemical, nuclear magnetic resonance (NMR), laboratory data, and symptoms and other information provided by the medical specialist (Table 1). An example of such training matrix with one output variable $(n=1)$ that may assume two possible values (positive or negative) is given in Fig. 4. 


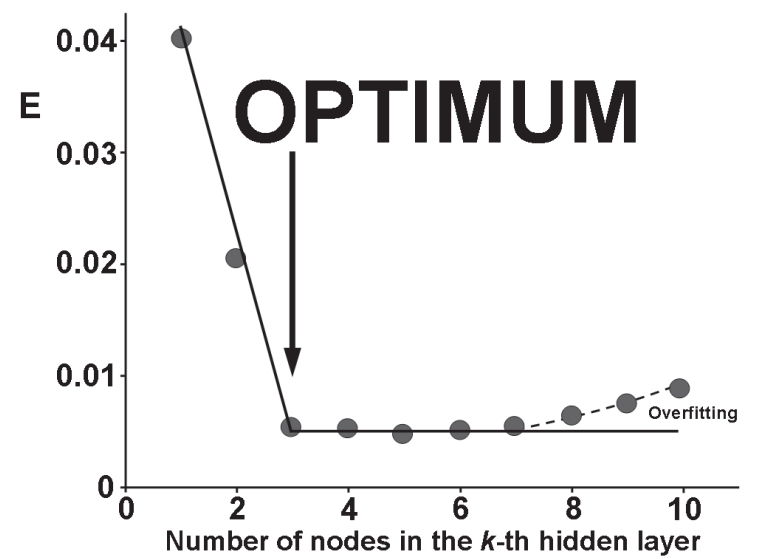

Fig. 5. Example of the plot used to select the optimal number of nodes in a given hidden layer. It is indicated that too high number of nodes might lead to overfitting.

Table 1. Brief overview of data in clinical context used as inputs for ANN.

\begin{tabular}{llll}
\hline Input data or method & Clinical context & Output information & Reference \\
\hline $\begin{array}{l}\text { Age, cholesterol } \\
\text { concentration, arterial } \\
\text { hypertension }\end{array}$ & Coronary artery disease & Diagnosis & (Atkov et al. 2012) \\
$\begin{array}{l}\text { Heart sound } \\
\text { Hematologic profile }\end{array}$ & Valve stenosis & Diagnosis & (Uğuz 2012) \\
$\begin{array}{l}\text { Visual information of } \\
\text { wireless capsule endoscopy }\end{array}$ & Small bowel tumors & $\begin{array}{l}\text { Diagnosis, classification of } \\
\text { tumor }\end{array}$ & (Barbosa et al. 2012) \\
$\begin{array}{l}\text { Glucose concentration }- \\
\text { Near-infrared spectroscopy }\end{array}$ & Diabetes & $\begin{array}{l}\text { Diagnosis } \\
\begin{array}{l}\text { Demographic and } \\
\text { clinicopathologic data, } \\
\text { surgical outcome }\end{array}\end{array}$ & Hepatocellular carcinoma \\
$\begin{array}{l}\text { Cytology of effusion fluid } \\
\text { Speech record }\end{array}$ & Carcinoma & $\begin{array}{l}\text { Prediction of disease free } \\
\text { survival }\end{array}$ & (Ho et al. 2012) \\
$\begin{array}{l}\text { Electroencephalographic } \\
\text { (EEG) recordings }\end{array}$ & Oral/Oropharyngeal cancer & $\begin{array}{l}\text { Detection of nasalence } \\
\text { (hypernasality) }\end{array}$ & (de Bruijn et al. 2011) \\
\hline
\end{tabular}

\section{OVERVIEW OF ANNS IN MEDICAL DIAGNOSIS}

There are several reviews concerning the application of ANNs in medical diagnosis. The concept was first outlined in 1988 in the pioneering work of (Szolovits et al. 1988) and since then many papers have been published. The general application of ANNs in medical diagnosis has previously been described
(Alkim et al. 2012). For example, ANNs have been applied in the diagnosis of: $(i)$ colorectal cancer (Spelt et al. 2012), (ii) multiple sclerosis lesions (Mortazavi et al. 2012a, b), (iii) colon cancer (Ahmed 2005), (iv) pancreatic disease (Bartosch-Härlid et al. 2008), (v) gynecological diseases (Siristatidis et al. 2010), and (vi) early diabetes (Shankaracharya et al. 2010). In addition, ANNs have also been applied in the analysis 
of data and diagnostic classification of patients with uninvestigated dyspepsia in gastroenterology (Pace and Savarino 2007) and in the search for biomarkers (Bradley 2012). A novel, general, fast, and adaptive disease diagnosis system has been developed based on learning vector quantization ANNs. This algorithm is the first proposed adaptive algorithm and can be applied to completely different diseases, as demonstrated by the $99.5 \%$ classification accuracy achieved for both breast and thyroid cancers. Cancer, diabetes, and cardiovascular diseases are among the most serious and diverse diseases. The amount of data coming from instrumental and clinical analysis of these diseases is quite large and therefore the development of tools to facilitate diagnosis is of great relevance. For this reason, we will provide a brief overview of the advances in the application of ANNs to the field of diagnosis for each of these diseases.

\section{Cardiovascular diseases}

Cardiovascular diseases (CVDs) are defined as all diseases that affect the heart or blood vessels, both arteries and veins. They are one of the most important causes of death in several countries. According to the National Center of Health Statistics (NCHS, www. cdc.gov/nchs), CVD represents the leading cause of death in the United States. CVD has therefore become an important field of study during the last 20 years.

Based on a bibliography search (ScienceDirect), more than one thousand papers about the use of ANNs in cardiovascular diseases and related topics have been published since 2008. According to the NCHS, coronary artery disease (CAD) is currently the leading cause of death worldwide, therefore early diagnosis is very important. With this aim, Karabulut and Ibrikçi applied ANNs with the Levenberg-Marquardt back propagation algorithm as base classifiers of the rotation forest ensemble method (Karabulut and Ibrikçi 2012). Diagnosis of CAD with 91.2\% accuracy was achieved from data collected non-invasively, cheaply, and easily from the patient. Other data such as age, different kinds of cholesterol, or arterial hypertension have been used to diagnose CAD (Atkov et al. 2012). The model that performed with the best accuracy (93\%) was the one that included both genetic and non-genetic factors related to the disease. Despite these promising results, it must be noted that for some models the accuracy was lower than $90 \%$. ANNs have also been applied in other heart diseases, such as heart valve defects (Uğuz 2012). Heart sound recordings were obtained from 120 subjects and classified as normal, pulmonary, and mitral stenosis heart valve diseases via stethoscopy. Correct classification was achieved for $95 \%$ of the different sounds. An average accuracy of $99.8 \%$ and $99.2 \%$ was achieved by two different models developed by (Özbay 2009) for the diagnosis of arrhythmias. Therefore, ANNs can be applied to the medical diagnosis of completely different diseases, for example detection of arrhythmias or coronary artery diseases, which are major causes of death worldwide. Classification accuracies higher than $90 \%$ are typically achieved, even exceeding $99 \%$ in some cases. As a result, ANNs have substantial potential in CVD diagnosis.

\section{Cancer}

According to the American Cancer Society (www. cancer.org), there will be more than 1.6 million newly diagnosed cases of cancer in the US in 2012. A rapid and correct diagnosis is essential for the clinical management of cancer, including selection of the most suitable therapeutic approach. The use of ANNs in distinguishing particular cancer types or the prediction of cancer development emerged in the late 1990s as a promising computational-based diagnostic tool using various inputs. Use of novel molecular approaches, such as micro-RNA screens, broadens the possibilities for the application of ANNs in the search for patterns specific for a certain disease, for example rectal cancer and its response to cytoreductive therapy (Kheirelseid et al. 2012). The application of neural networks trained on defined data sets was evaluated in 1994 for breast and ovarian cancer, opening a discussion on the suitability of particular data as inputs for ANN analysis, for example demographic (age), radiological (NMR), oncologic (tumor markers CA 15-3 or CA 125), and biochemical (albumin, cholesterol, high-density lipoprotein cholesterol, triglyceride, apolipoproteins A1 and B) data (Wilding et al. 1994). Later on, reasonable prediction of the measured in vitro chemotherapeutic response based on ${ }^{1} \mathrm{H}$ NMR of glioma biopsy extracts was achieved using ANNs to obtain automatic differential diagnosis of glioma (El-Deredy et al. 1997).

Moreover, advanced analytical methods, such as mass spectrometry, can offer suitable information for clinically relevant ANN analysis. This technique has recently been applied successfully in the diagnosis of ovarian cancer. Upon identification of the most informative points of the mass spectrum curve, for example by data mining, the integrated feed-forward ANN classifier showed an overall sensitivity of $98 \%$ and a specificity of $96 \%$, surpassing standard statistical methods such as Student's t-test (Thakur et al. 2011).

The applications of ANNs in radiology aim to develop automated decision support systems, assisting, for example, in the classification of brain tumors by magnetic resonance (Tate et al. 2006). The 
concept and design raised by Tate was also applied by Brougham and colleagues on lung carcinoma (Brougham et al. 2011). In this process, the same experimental protocol was carried out by two different teams of researchers examining two sets of whole-cell ${ }^{1} \mathrm{H}$ NMR spectra at different times. Whole-cell ${ }^{1} \mathrm{H}$ NMR spectra were recorded for two groups of human lung carcinoma cell lines, which were grown in culture and included $(i)$ the parent cell line DLKP, a human squamous non-small cell lung carcinoma; (ii) DLKP-A and (iii) DLKP-A5F, two resistant daughter lines; and (iv) A549 a human lung adenocarcinoma cell line. Despite demonstrated operator-induced sources of variation in the network, the ANN was able to classify the cell line correctly in $100 \%$ of cases independent of the spectra selected for training and validation. Therefore, the power of ANNs for the classification of different types of lung carcinoma in real clinical or laboratory situations was demonstrated. In summary, ANNs have been shown to use various primary data, ranging from clinical parameters to biochemical values, and provide increased diagnostic accuracy for various kinds of cancers.

\section{Diabetes}

Diabetes represents a serious health problem in developed countries, with estimated numbers reaching 366 million diabetes cases globally in 2030 (Leon et al. 2012). The most common type of diabetes is type II, in which the cellular response to insulin is impaired leading to disruption of tissue homeostasis and hyperglycemia. The standard in diabetes diagnosis or monitoring is direct measurement of glucose concentration in blood samples. Noninvasive methods based on near-infrared or Raman spectroscopy to monitor glucose levels were developed in 1992 (Arnold 1996) and nowadays are even available as a smartphone application. The ANNs extrapolate glucose concentrations from spectral curve, thus enabling convenient monitoring of diabetes during daily activities.

Quality of life itself, including satisfaction, social interactions, and depression, has been defined as an integral outcome measure in management of diabetes mellitus. Recently, Narasingarao et al developed a prototype neural network model to measure the quality of life in diabetic patients (Narasingarao et al. 2009). For this particular purpose, biological or biographical variables such as age, gender, weight, or fasting plasma glucose were used as input data. The results were comparable with those from traditional statistical methods. In 1997, simulation studies on neural predictive control of glucose concentration in subcutaneous tissue were carried out (Trajanoski et al. 1998). As a control strategy, Trajanoski and colleagues combined ANNs and nonlinear model predictive control. This approach allowed for large noise levels and time delays. However, administration of insulin or analogues was found to be unsuitable for rapid control and was adequate only for controlling slow perturbations. Later, a new control system called a neuro-fuzzy control system, which uses fuzzy logic principles and neural networks employing a minimal number of input data for correct output, was developed (Dazzi et al. 2001). This system is especially suitable for direct control of critically ill diabetic patients on intravenous nutrition and reduced the need for increased blood glucose testing and even the risk of hypoglycemia. Blood glucose level has also been monitored using inverse optimal neural control as a preliminary study in patients with type I diabetes (Leon et al. 2012). Recurrent neural networks were used to control the level of glucose and insulin following a desired trajectory (normal glucose absorption of a healthy person) to avoid hypoglycemia and hyperglycemia. ANNs have not only been applied in the tracking of glucose, but also in the diagnosis of diabetes. A neural network-based diagnostic system was developed by (Chan et al. 2011) with the aim of predicting fatal hypoglycemia episodes in type I diabetes patients. The data were collected from a cohort of 420 patients and included: (i) physiological parameters, (ii) rate of change of heart rate, (iii) corrected QT interval of electrocardiogram signal, and (iv) rate of change of corrected QT interval. Data from 320 patients were used to train the network and the remainder for its verification. A sensitivity of $79.30 \%$ and specificity of $60.53 \%$ was achieved, better values than those obtained using other approaches such as statistical or fuzzy regression methods. In conclusion, the use of ANNs in the diagnosis of diabetes provides an improvement in accuracy, sensitivity, and specificity in comparison with other methods, thus contributing to improved clinical management of diabetes mellitus.

\section{FUNDAMENTAL STEPS IN ANNS-BASED MEDICAL DIAGNOSIS}

The workflow of ANN analysis arising from the outlined clinical situations is shown in Fig. 6 which provides a brief overview of the fundamental steps that should be followed to apply ANNs for the purposes of medical diagnosis with sufficient confidence. 


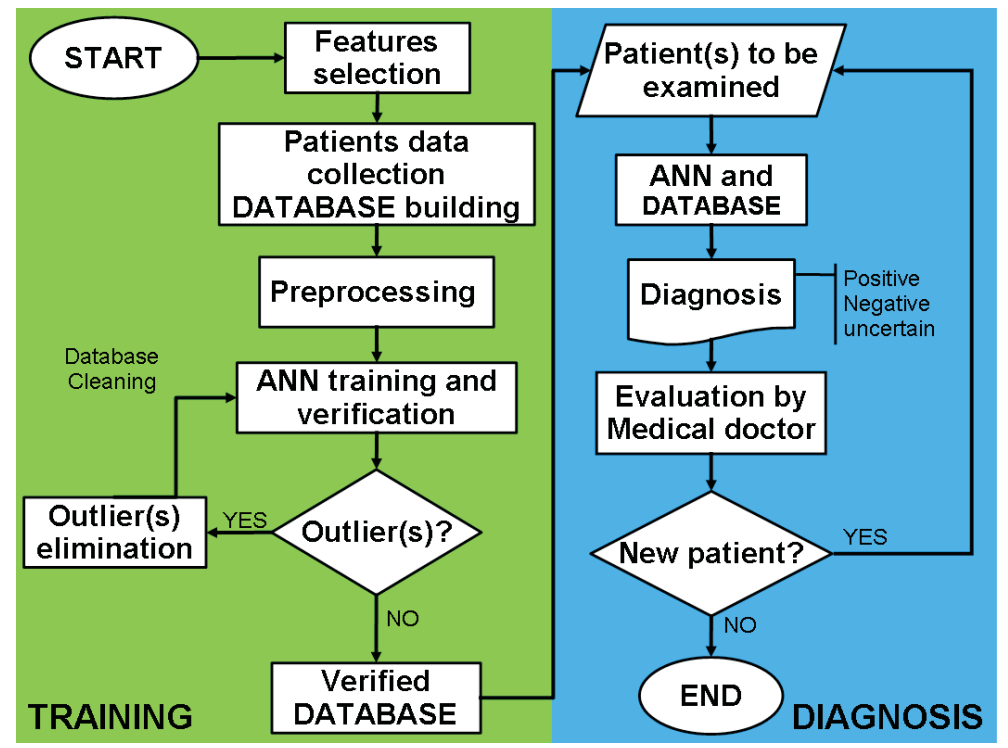

Fig. 6. Diagram of fundamental steps in ANNs-based medical diagnosis. Building of the database and "learning" represents the left half (green) and its application for the diagnosis is the right part (blue).

For the reasons discussed above, the network receives patient's data to predict the diagnosis of a certain disease. After the target disease is established, the next step is to properly select the features (e.g., symptoms, laboratory, and instrumental data) that provide the information needed to discriminate the different health conditions of the patient. This can be done in various ways. Tools used in chemometrics allow the elimination of factors that provide only redundant information or those that contribute only to the noise. Therefore, careful selection of suitable features must be carried out in the first stage. In the next step, the database is built, validated and "cleaned" of outliers. After training and verification, the network can be used in practice to predict the diagnosis. Finally, the predicted diagnosis is evaluated by a clinical specialist. The major steps can be summarized as:

\section{Features selection}

Building the database

Data cleaning and preprocessing

Data homoscedasticity

Training and verification of database using ANN

Network type and architecture

Training algorithm

Verification

Robustness of ANN-based approaches

Testing in medical practice
The individual steps listed above will be shortly commented and some details given.

\section{Features selection}

Correct diagnosis of any disease is based on various, and usually incoherent, data (features): for example, clinicopathologic evaluation, laboratory and instrumental data, subjective anamnesis of the patient, and considerations of the clinician. Clinicians are trained to extract the relevant information from each type of data to identify possible diagnoses. In artificial neural network application such data are called "features". Features can be symptoms, biochemical analysis data and/or whichever other relevant information helping in diagnosis. Therefore, the experience of the professional is closely related to the final diagnosis. The ability of ANNs to learn from examples makes them very flexible and powerful tools to accelerate medical diagnosis. Some types of neural networks are suitable for solving perceptual problems while others are more adapted for data modeling and functional approximation (Dayhoff and Deleo 2001). Regardless of the features selected, those chosen for training the neural network should be "robust" indicators for a given clinical situation or pathology. In general, feature selection relies upon previous clinical experience. Features that bring insufficient, redundant, non-specific, or noisy information about the investigated problem should be avoided. The selection/extraction of suitable features among all 
available ones is usually carried out using various approaches. The most important and best-known tools for variable selection are powerful mathematical means of data mining such as principal components analysis, genetic algorithm (Yan et al. 2008), or ANNs (Verikas and Bacauskiene 2002).

\section{Building the database}

The neural network is trained using a suitable database of "example" cases. An "example" is provided by one patient whose values for the selected features have been collected and evaluated. The quality of training and the resultant generalization, and therefore the prediction ability of the network, strongly depend on the database used for the training. The database should contain a sufficient number of reliable "examples" (for which the diagnosis is known) to allow the network to learn by extracting the structure hidden in the dataset and then use this "knowledge" to "generalize" the rule to new cases. In addition, clinical laboratory data should be in a form that is readily transferable to programs for computer-aided diagnosis (Strike et al. 1986).

\section{Data cleaning and preprocessing}

Data in the training database must be preprocessed before evaluation by the neural network. Several approaches are available for this purpose. Data are normally scaled to lie within the interval $[0,1]$ because the most commonly used transference function is the socalled logistic one. In addition, it has been demonstrated that cases for which some data are missing should be removed from the database to improve the classification performance of the network (Gannous and Elhaddad 2011). A decrease in the classification performance of the network is observed for imbalanced databases (those with a different number of cases for each class) (Mazurowski et al. 2008).

\section{Data homoscedasticity}

Once the suitable features, database, data preprocessing method, training algorithm, and network architecture have been identified, data concerning "new" patients who are not included in the training database can be evaluated by the trained network. The question asked is whether the new data belong to the same population as those in the database (homoscedasticity). Failure at this step might lead the network to misclassify the new data. This problem can be solved by the use of an additional parameter that indicates the population to which a certain sample belongs.

\section{Training and verification of database using ANN}

Network type and architecture

Although multilayer feed-forward neural networks are most often used, there are a large variety of other networks including bayesian, stochastic, recurrent, or fuzzy. The optimal neural network architecture must be selected in the first stage. This is usually done testing networks with different number of hidden layers and nodes therein. The optimal architecture is that for which the minimum value of $E$ (Eq. 4) for both training and verification is obtained.

\section{Training algorithm}

Various training algorithms are available. However, the most commonly used is back propagation (Zupan and Gasteiger 1999; Ahmed 2005). As discussed in "Network learning" section, backpropagation algorithm requires the use of two training parameters: (i) learning rate and (ii) momentum. Usually, high values of such parameters lead to unstable learning, and therefore poor generalization ability of the network. The optimal values of the training parameters depend upon the complexity of the studied system. In general, the value of momentum is lower than that of learning rate. In addition, the sum of their values should be approximately equal to one.

\section{Verification}

ANNs-based medical diagnosis should be verified by means of a dataset different from that one used for training.

\section{Robustness of ANN-based approaches}

It is well known that ANNs are able to tolerate a certain level of noise in the data and consequently they typically provide sufficient prediction accuracy. However, this noise might sometimes cause misleading results, especially when modeling very complex systems such as the health condition of a human body. Such noise would not only impact the normal uncertainty of the measured data but might also impact secondary factors, for example the coexistence of more than one disease. Crossed effects cannot be predicted unless they have been considered during building of the training database. Any factor that influences the symptoms of the disease under study must be taken into account by including such cases in the database. Only in this way can the network correctly classify the patient. Of course, one way to avoid this is to combine the experience of the clinical specialist with the discriminative power of ANN-based approaches.

\section{Testing in medical practice}

As the final step in ANN-aided diagnosis should be testing in medical practice. For each new patient the network's outcome is to be carefully examined by a clinician. Medical data of patients for which the predicted diagnosis is correct can be eventually included in the training database. 
However, wide and extensive evaluation of ANNaided diagnosis applications in clinical setting is necessary even throughout different institutions. Verified ANN-aided medical diagnosis support applications in clinical setting are necessary condition for further expansion in medicine.

\section{CONCLUSIONS}

ANNs represent a powerful tool to help physicians perform diagnosis and other enforcements. In this regard, ANNs have several advantages including:

(i) The ability to process large amount of data

(ii) Reduced likelihood of overlooking relevant information

(iii) Reduction of diagnosis time

ANNs have proven suitable for satisfactory diagnosis of various diseases. In addition, their use makes the diagnosis more reliable and therefore increases patient satisfaction. However, despite their wide application in modern diagnosis, they must be considered only as a tool to facilitate the final decision of a clinician, who is ultimately responsible for critical evaluation of the ANN output. Methods of summarizing and elaborating on informative and intelligent data are continuously improving and can contribute greatly to effective, precise, and swift medical diagnosis.

\section{ACKNOWLEDGEMENTS}

Support from Ministry of Education, Youth and Sports of the Czech Republic (Projects MSM0021622411, MSM0021627501, MSM0021622430, CZ.1.05/1.1.00/ 02.0123 and HistoPARK CZ.1.07/2.3.00/20.0185), and the Czech Science Foundation (Projects No. $104 / 08 / 0229,202 / 07 / 1669)$ is acknowledged. This research was also supported by CEPLANT, the project $\mathrm{R} \& \mathrm{D}$ center for low-cost plasma and nanotechnology surface modifications (CZ.1.05/2.1.00/03.0086 funding from the European Regional Development Fund). E. M. Peña-Méndez thanks the University of La Laguna for partial support.

\section{REFERENCES}

Ahmed F. Artificial neural networks for diagnosis and survival prediction in colon cancer. Mol Cancer. 4: $29,2005$.
Aleksander I, Morton H. An introduction to neural computing. Int Thomson Comput Press, London 1995.

Alkim E, Gürbüz E, Kiliç E. A fast and adaptive automated disease diagnosis method with an innovative neural network model. Neur Networks. 33: 88-96, 2012.

Amato F, González-Hernández J, Havel J. Artificial neural networks combined with experimental design: a "soft" approach for chemical kinetics. Talanta. 93: 72-78, 2012.

Arnold M. Non-invasive glucose monitoring. Curr Opin Biotech. 7: 46-49, 1996.

Atkov O, Gorokhova S, Sboev A, Generozov E, Muraseyeva E, Moroshkina S and Cherniy N. Coronary heart disease diagnosis by artificial neural networks including genetic polymorphisms and clinical parameters. J Cardiol. 59: 190-194, 2012.

Barbosa D, Roupar D, Ramos J, Tavares A and Lima C. Automatic small bowel tumor diagnosis by using multi-scale wavelet-based analysis in wireless capsule endoscopy images. Biomed Eng Online. 11: 3, 2012.

Bartosch-Härlid A, Andersson B, Aho U, Nilsson J, Andersson R. Artificial neural networks in pancreatic disease. Br J Surg. 95: 817-826, 2008.

Barwad A, Dey P, Susheilia S. Artificial neural network in diagnosis of metastatic carcinoma in effusion cytology. Cytometry B Clyn Cytom. 82: 107-111, 2012.

Basheer I, Hajmeer M. Artificial neural networks: fundamentals, computing, design, and application. J Microbiol Meth. 43: 3-31, 2000.

Bradley B. Finding biomarkers is getting easier. Ecotoxicology. 21: 631-636, 2012.

Brougham D, Ivanova G, Gottschalk M, Collins D, Eustace A, O'Connor R, Havel J. Artificial neural networks for classification in metabolomic studies of whole cells using $1 \mathrm{H}$ nuclear magnetic resonance. J Biomed Biotechnol. 2011: 158094, 2011.

Catalogna M, Cohen E, Fishman S, Halpern Z, Nevo U, Ben-Jacob E. Artificial neural networks based controller for glucose monitoring during clamp test. PloS One. 7: e44587, 2012.

Chan K, Ling S, Dillon T, Nguyen H. Diagnosis of hypoglycemic episodes using a neural network based rule discovery system. Expert Syst Appl. 38: 9799-9808, 2011.

Dayhoff J, Deleo J. Artificial Neur Networks: Opening the Black Box. Cancer. 91: 1615-1635, 2001.

Dazzi D, Taddei F, Gavarini A, Uggeri E, Negro R, Pezzarossa A. The control of blood glucose in the critical diabetic patient: a neuro-fuzzy method. J Diabet Complicat. 15: 80-87, 2001. 
de Bruijn M, ten Bosch L, Kuik D, Langendijk J, Leemans C, Verdonck-de Leeuw I. Artificial neural network analysis to assess hypernasality in patients treated for oral or oropharyngeal cancer. Logoped Phoniatr Vocol. 36: 168-174, 2011.

Dey P, Lamba A, Kumari S, Marwaha N. Application of an artificial neural network in the prognosis of chronic myeloid leukemia. Anal Quant Cytol Histol. 33: 335-339, 2012.

El-Deredy W, Ashmore S, Branston N, Darling J, Williams S, Thomas D. Pretreatment prediction of the chemotherapeutic response of human glioma cell cultures using nuclear magnetic resonance spectroscopy and artificial neural networks Cancer Res. 57: 4196-4199, 1997.

Elveren E, Yumuşak N. Tuberculosis disease diagnosis using artificial neural network trained with genetic algorithm. J Med Syst. 35: 329-332, 2011.

Er O, Temurtas F, Tanrıkulu A. Tuberculosis Disease Diagnosis Using Artificial Neural Networks. J Med Syst. 34: 299-302, 2008.

Fedor P, Malenovsky I, Vanhara J, Sierka W, Havel J. Thrips (Thysanoptera) identification using artificial neural networks. Bull Entomol Res. 98: 437-447, 2008.

Fernandez de Canete J, Gonzalez-Perez S, Ramos-Diaz JC. Artificial neural networks for closed loop control of $i$ silico and ad hoc type 1 diabetes. Comput Meth Progr Biomed. 106: 55-66, 2012.

Fernandez-Blanco E, Rivero D, Rabunal J, Dorado J, Pazos A, Munteanu C. Automatic seizure detection based on star graph topological indices. J Neurosci Methods. 209: 410-419, 2012.

Gannous AS, Elhaddad YR. Improving an Artificial Neural Network Model to Predict Thyroid Bending Protein Diagnosis Using Preprocessing Techniques. WASET. 50: 124-128, 2011.

Havel J, Peña E, Rojas-Hernández A, Doucet J, Panaye A. Neural networks for optimization of highperformance capillary zone electrophoresis methods. J Chromatogr A. 793: 317-329, 1998.

Ho W-H, Lee K-T, Chen H-Y, Ho T-W, Chiu H-C. Disease-free survival after hepatic resection in hepatocellular carcinoma patients: a prediction approach using artificial neural network. PloS One. 7: e29179, 2012.

Karabulut E, Ibrikçi T. Effective diagnosis of coronary artery disease using the rotation forest ensemble method. J Med Syst. 36: 3011-3018, 2012.

Kheirelseid E, Miller N, Chang K, Curran C, Hennessey E, Sheehan M, Newell J, Lemetre C, Balls G, Kerin M. miRNA expressions in rectal cancer as predictors of response to neoadjuvant chemoradiation therapy. Int J Colorectal Dis. 2012.
Leon BS, Alanis AY, Sanchez E, Ornelas-Tellez F, RuizVelazquez E. Inverse optimal neural control of blood glucose level for type 1 diabetes mellitus patients. J Franklin I. 349: 1851-1870, 2012.

Li Y, Rauth AM, Wu XY. Prediction of kinetics of doxorubicin release from sulfopropyl dextran ion-exchange microspheres using artificial neural networks. Eur J Pharm Sci. 24: 401-410, 2005.

Mazurowski M, Habas P, Zurada J, Lo J, Baker J, Tourassi G. Training neural network classifiers for medical decision making: the effects of imbalanced datasets on classification performance. Neural networks. 21: 427-436, 2008.

Michalkova V, Valigurova A, Dindo M, Vanhara J. Larval morphology and anatomy of the parasitoid Exorista larvarum (Diptera: Tachinidae), with an emphasis on cephalopharyngeal skeleton and digestive tract. J Parasitol. 95: 544-554, 2009.

Molga E, van Woezik B, Westerterp K. Neural networks for modelling of chemical reaction systems with complex kinetics: oxidation of 2-octanol with nitric acid. Chem Eng Process. 39: 323-334, 2000.

Mortazavi D, Kouzani A, Soltanian-Zadeh H. Segmentation of multiple sclerosis lesions in MR images: a review. Neuroradiology. 54: 299-320, 2012a.

Mortazavi D, Kouzani AZ, Soltanian-Zadeh H. Segmentation of multiple sclerosis lesions in MR images: a review. Neuroradiology. 54: 299-320, 2012b.

Murarikova N, Vanhara J, Tothova A, Havel J. Polyphasic approach applying artificial neural networks, molecular analysis and postabdomen morphology to West Palaearctic Tachina spp. (Diptera, Tachinidae). Bull Entomol Res. 101: 165-175, 2010.

Narasingarao M, Manda R, Sridhar G, Madhu K, Rao A. A clinical decision support system using multilayer perceptron neural network to assess well being in diabetes. J Assoc Physicians India. 57: 127-133, 2009.

Özbay Y. A new approach to detection of ECG arrhythmias: Complex discrete wavelet transform based complex valued artificial neural network. J Med Syst. 33: 435-445, 2009.

Pace F, Savarino V. The use of artificial neural network in gastroenterology: the experience of the first 10 years. Eur J Gastroenterol Hepatol. 19: 1043-1045, 2007.

Rodríguez Galdón B, Peña-Méndez E, Havel J, Rodríguez Rodríguez E, Díaz Romero C. Cluster Analysis and Artificial Neural Networks Multivariate Classification of Onion Varieties. J Agric Food Chem: 11435-11440, 2010.

Saghiri M, Asgar K, Boukani K, Lotfi M, Aghili H, Delvarani A, Karamifar K, Saghiri A, Mehrvarzfar P, 
Garcia-Godoy F. A new approach for locating the minor apical foramen using an artificial neural network. Int Endod J. 45: 257-265, 2012.

Shankaracharya, Odedra D, Samanta S, Vidyarthi A. Computational intelligence in early diabetes diagnosis: a review. Rev Diabet Stud. 7: 252-262, 2010.

Siristatidis C, Chrelias C, Pouliakis A, Katsimanis E, Kassanos D. Artificial neural networks in gyneacological diseases: Current and potential future applications. Med Sci Monit. 16: 231-236, 2010.

Spelt L, Andersson B, Nilsson J, Andersson R. Prognostic models for outcome following liver resection for colorectal cancer metastases: A systematic review. Eur J Surg Oncol. 38: 16-24, 2012.

Strike P, Michaeloudis A, Green AJ. Standardizing clinical laboratory data for the development of transferable computer-based diagnostic programs. Clin Chem. 32: 22-29, 1986.

Szolovits P, Patil RS, Schwartz W. Artificial Intelligence in Medical Diagnosis. Ann Intern Med. 108: 80-87, 1988.

Tate A, Underwood J, Acosta D, Julià-Sapé M, Majós C, Moreno-Torres A, Howe F, van der Graaf M, Lefournier V, Murphy M, Loosemore A, Ladroue $\mathrm{C}$ et al. Development of a decision support system for diagnosis and grading of brain tumours using in vivo magnetic resonance single voxel spectra. NMR Biomed. 19: 411-434, 2006.

Thakur A, Mishra V, Jain S. Feed forward artificial neural network: tool for early detection of ovarian cancer. Sci Pharm. 79: 493-505, 2011.

Trajanoski Z, Regittnig W, Wach P. Simulation studies on neural predictive control of glucose using the subcutaneous route. Comput Meth Progr Biomed. 56: 133-139, 1998.

Uğuz H. A biomedical system based on artificial neural network and principal component analysis for diagnosis of the heart valve diseases. J Med Syst. 36: 61-72, 2012.

Verikas A, Bacauskiene M. Feature selection with neural networks. Pattern Recogn Lett. 23: 1323 1335, 2002.

Wilding P, Morgan M, Grygotis A, Shoffner M, Rosato E. Application of backpropagation neural networks to diagnosis of breast and ovarian cancer. Cancer Lett. 77: 145-153, 1994.

Yan H, Zheng J, Jiang Y, Peng C, Xiao S. Selecting critical clinical features for heart diseases diagnosis with a real-coded genetic algorithm. Appl Soft Comput. 8: 1105-1111, 2008.

Zupan J, Gasteiger J. Neural networks in chemistry and drug design. Wiley VCH, Weinheim, 380 p. 1999. 